department of Peking University First Hospital from January 2007 to May 2016. The primary causes of death were identified, the standardised mortality ratio (SMR) and years of life lost (YLL) were calculated based on the National Bureau of Statistics of China for the general population, the survival in the first decade was performed using Kaplan-Meier analysis, and the predictors of mortality were evaluated by multivariable cox regression.

Results: A total of 226 DM and 54 PM cases were included and the mean age of onset was $49.9 \pm 14.8$ years for DM and $48.1 \pm 17.1$ years for PM. The median follow-up duration was 40.6 (11.6-77.6) months. Among 267 patients who were successfully traced, 66 patients died. Infection $(50.0 \%)$ was the leading cause of death followed by malignancy (19.7\%), and interstitial lung disease (ILD) (9.1\%). The overall age and sex adjusted SMR was 9.0 (95\% Cl 6.8-11.2) for DM, and $5.0(95 \% \mathrm{Cl} 2.4-7.5)$ for PM. The overall age and sex adjusted SMR of DM/PM patients with ILD was $8.4(95 \% \mathrm{Cl} 5.8-11.0)$, and the SMR of the patients with malignancy was $14.9(95 \% \mathrm{Cl} 8.5-21.2)$. The YLL of women and men were 37.5 and 28.4 years respectively for DM, and 24.3 and 12.0 years respectively for PM (Table1). The 10-year survival of patients with ILD or malignancy was significantly worse than those without ILD or malignancy respectively (Figure 1 and 2). The independent predictors of mortality for DM were age of disease onset, respiratory muscle involvement and malignancy; and the independent predictor of mortality for PM was age at disease onset (Table2).

Table 1. The standardized mortality ratio (SMR), life expectancy (LE) and years of life lost (YLL)

\begin{tabular}{lccc}
\hline & Overall $(\mathrm{n}=280)$ & Females $(\mathrm{n}=201)$ & Males $(\mathrm{n}=79)$ \\
\hline Dermatomyositis & 226 & 163 & 63 \\
Death number & 55 & 37 & 18 \\
SMR (95\% Cl) & $9.0(6.8-11.2)$ & $12.0(6.8-11.2)$ & $6.0(3.2-8.7)$ \\
LE of general population (years) & - & 80.8 & 75.8 \\
LE of DM patients (years) & - & 43.3 & 47.4 \\
YLL (years) & - & 37.58 & 28.4 \\
Polymyositis & 54 & 38 & 16 \\
Death number & 11 & 8 & 3 \\
SMR (95\% Cl) & $5.0(2.4-7.5)$ & $4.2(1.3-7.2)$ & $9.3(-1.2-19.8)$ \\
LE of general population (years) & - & 80.8 & 75.8 \\
LE of PM patients (years) & - & 56.5 & 63.8 \\
YLL (years) & - & 24.3 & 12.0 \\
\hline
\end{tabular}

Abbreviations: DM: dermatomyositis; SMR: standardised mortality ratio; $\mathrm{Cl}$ : confidence interval; LE: life expectancy; YLL: years of life lost; PM: polymyositis.

Table 2. Multivariable cox regression analyses of risk factors in the DM/PM patients

\begin{tabular}{lccc}
\hline Variables & $\mathrm{HR}$ & $95 \% \mathrm{Cl}$ & $P$ value \\
\hline Dermatomyositis & & & \\
$\quad$ Age $^{\star}$ & 1.04 & $1.01-1.06$ & $<0.001$ \\
$\quad$ ILD & 1.35 & $0.74-2.48$ & 0.319 \\
Respiratory muscle involvement & 2.58 & $1.19-5.58$ & 0.016 \\
Malignancy & 3.12 & $1.49-6.58$ & 0.003 \\
Polymyositis & & & \\
$\quad$ Age $^{*}$ & 1.08 & $1.00-1.16$ & 0.044 \\
ILD & 2.47 & $0.18-34.00$ & 0.500 \\
ESR & 1.02 & $0.99-1.04$ & 0.174 \\
\hline
\end{tabular}

*Age: Age at disease onset. Abbreviations: HR: Hazard Ratio; Cl: confidence interval; ILD: interstitial lung disease; ESR: erythrocyte sedimentation rate.

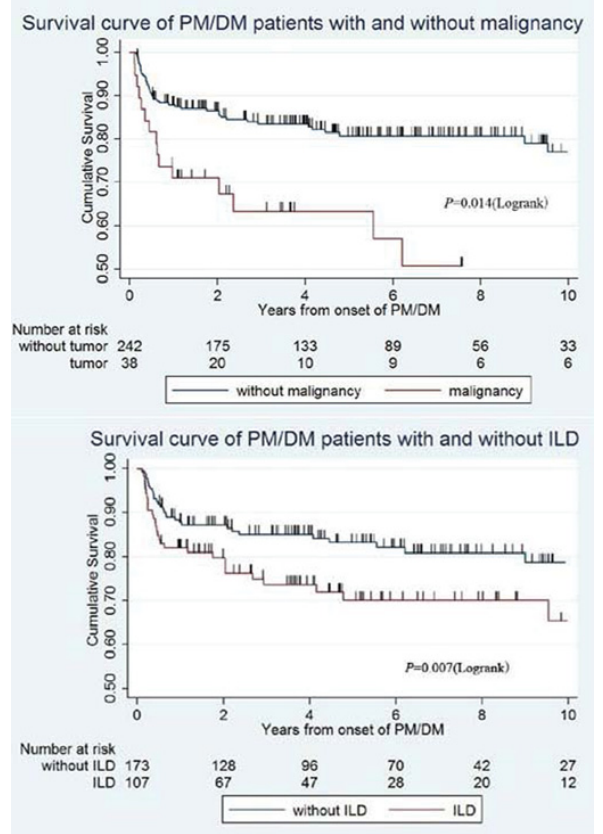

Conclusions: Mortality of DM/PM patients in China is substantial, especially in females, and those with ILD or malignancy. Infection was the leading cause of death. Patients with older age at onset, respiratory muscle involvement, ILD, and malignancy need to be paid more attention.

Disclosure of Interest: None declared

DOI: 10.1136/annrheumdis-2017-eular.2873

\section{OP0126 A PHASE 2 STUDY OF SAFETY AND EFFICACY OF ANABASUM (JBT-101) IN SYSTEMIC SCLEROSIS}

R. Spiera ${ }^{1}$, L. Hummers ${ }^{2}$, L. Chung ${ }^{3}$, T. Frech ${ }^{4}$, R. Domsic ${ }^{5}$, D. Furst ${ }^{6}$

J. Gordon ${ }^{1}$, M. Mayes ${ }^{7}$, R. Simms ${ }^{8}$, S. Constantine ${ }^{9}$, B. White ${ }^{9} .{ }^{1}$ Hospital for Special Surgery, New York City; ${ }^{2}$ Johns Hopkins, Baltimore; ${ }^{3}$ Stanford, Palo Alto;

${ }^{4}$ University of Utah, Salt Lake City; ${ }^{5}$ University of Pittsburgh, Pittsburgh; ${ }^{6}$ Arthritis Association of Southern California, Los Angeles; ${ }^{7}$ University of Texas, Houston;

${ }^{8}$ Boston University, Boston: ${ }^{9}$ Corbus Pharmaceuticals, Inc., Norwood, United States

Background: Anabasum (JBT-101) is a synthetic, oral, non-immunosuppressive, preferential CB2 agonist. It inhibits onset and activates resolution of innate immune responses in animal models of systemic sclerosis (SSc).

Objectives: Evaluate safety and efficacy of anabasum in SSc

Methods: A double-blind, randomized, placebo (PBO)-controlled Phase 2 trial dosed 42 diffuse cutaneous SSc subjects with disease duration $\leq 6$ years on stable medication including immunosuppressive drugs. Subjects received anabasum 5 $\mathrm{mg}$ QD, $20 \mathrm{mg} \mathrm{QD}$, or $20 \mathrm{mg}$ BID on Days 1-28, then $20 \mathrm{mg}$ BID on Days 29-84, or PBO on Days 1-84. Subjects were followed off study drug on Days $85-113$. The primary safety outcome was treatment-emergent adverse events (TEAEs). The primary efficacy outcome was improvement in ACR Combined Response Index in diffuse cutaneous Systemic Sclerosis (CRISS) score, combined anabasum group vs PBO, Days 29-113 (end of Weeks 4-16). The five domains of the ACR CRISS are the modified Rodnan skin score, HAQ-DI, patient and physician global assessments, and FVC \% predicted.

Results: Of 42 dosed subjects, $27(64 \%)$ received anabasum and $15(36 \%)$ received PBO. Three anabasum subjects withdrew: $1(3.7 \%)$ for a TEAE of moderate dizziness; 1 withdrew consent; and 1 by physician decision. One PBO subject withdrew consent. Baseline demographic and CRISS domain scores were similar except slightly more anabasum subjects used background immunosuppressive drugs (93\% versus $80 \%$, anabasum vs $\mathrm{PBO}$ ). Seventeen $(63 \%)$ anabasum subjects had 66 TEAEs, and $9(60 \%)$ PBO subjects had 35 TEAEs. There were no serious, severe, or unexpected TEAEs related to anabasum. Severity and relationship of TEAEs to study drug were similar in both groups. The most frequent TEAEs by MedDRA system (\% anabasum vs $\%$ PBO) were: nervous system ( $37 \%$ vs $27 \%)$; general disorders $(30 \%$ vs $7 \%)$; gastrointestinal $(22 \%$ vs $20 \%)$; infections (22\% vs $20 \%)$; musculoskeletal (22\% vs $13 \%$ ); and investigations ( $0 \%$ vs $20 \%$ ). The most frequent TEAEs in anabasum subjects were dizziness $(22 \%)$ and fatigue $(19 \%)$ which were usually mild. Anabasum subjects had greater improvement in ACR CRISS scores than PBO subjects (mixed model repeated measures analysis, $p=0.044,1$-sided). The median ACR CRISS scores at the end of Weeks $4,8,12$, and 16 (anabasum vs PBO) were $3.0 \%$ vs $1.0 \%, 19.0 \%$ vs $1.0 \%, 27.5 \%$ vs $1.0 \%$, and $33.0 \%$ vs $1.0 \%$, respectively. Among anabasum subjects, $\sim 50 \%$ had ACR CRISS $\geq 20 \%$ after 8 weeks of dosing. The individual domains of the ACR CRISS score showed greater improvement, improvement that reached minimal important differences in several domains, and less worsening in anabasum vs PBO groups. Anabasum subjects had greater improvement in SSc skin symptoms and itch. Plasma metabolipidomic profiles showed anabasum, not PBO, shifted lipid mediator production to increase pro-resolving vs pro-inflammatory lipid mediators.

Conclusions: Anabasum provided significant and medically meaningful efficacy in SSc as assessed by the ACR CRISS score and its individual domains and had acceptable safety and tolerability in this Phase 2 trial. These data support continued clinical development of anabasum for the treatment of SSc.

Disclosure of Interest: R. Spiera: None declared, L. Hummers: None declared, L. Chung: None declared, T. Frech: None declared, R. Domsic: None declared, D. Furst: None declared, J. Gordon: None declared, M. Mayes: None declared, R. Simms: None declared, S. Constantine Employee of: Corbus Pharmaceuticals, Inc., B. White Employee of: Corbus Pharmaceuticals, Inc.

DOI: 10.1136/annrheumdis-2017-eular.2712

\section{OP0127 TEN YEARS FOLLOW-UP OF GASTROINTESTINAL INVOLVEMENT BY THE SMALL INTESTINAL CLEARANCE IN PATIENTS WITH SYSTEMIC SCLEROSIS}

N. Hashimoto ${ }^{1}$, M. Kitano ${ }^{2}$, T. Nakazawa ${ }^{3}$, T. Iwasaki ${ }^{4}$, T. Hashimoto ${ }^{1}$ ${ }^{1}$ Hashimoto Rheumatology Clinic, Osaka; ${ }^{2}$ Division of Rheumatology, Department of Internal Medicine, Hyogo College of Medicine, Nishinomiya; ${ }^{3}$ Osaka Saiseikai Nakatsu Hospital, Osaka; ${ }^{4}$ Department of Pharmacy, Hyogo University of Health Sciences, Kobe, Japan

Background: Systemic sclerosis (SSc) is an inflammatory autoimmune disease characterized by fibrosis and small vascular involvement in the skin, lungs, heart and gastrointestinal (GI) tract. The esophagus is the most frequently involved GI tract disorder. Although the small intestinal involvement such as malabsorption and pseudo-obstruction is less common, it has been related to morbidity and mortality of SSc patients. We previously reported a close correlation between the 
esophageal diameter and the small intestinal clearance (SIC) (EULAR 2011). We also suggested that anti-centromere antibodies (ACA) are an important factor of esophageal dilatation in SSc patients (EULAR 2010). However, changes of small intestinal involvement during long-term follow-up have not yet been defined.

Objectives: The aim of this study was to evaluate a correlation between the small intestinal involvement and clinical features in SSc patients during ten years follow-up.

Methods: Fifty-five patients with a definite diagnosis of SSc [52 female and 3 male with mean age of 59.4 years (range 29-77 years)] were included in the study. Thirteen (23.6\%) patients were classified as diffuse SSc and $42(76.4 \%)$ as limited SSc. The SIC grade was classified according to barium meal reach at 30 min after intake; grade $1(>2 / 3$ of the whole small intestine), grade $2(1 / 3 \sim 2 / 3)$, grade $3(<1 / 3)$, grade 4 (the duodenum). The SIC change was classified as follows; grade 1 and 2 or grade non-increase during follow-up was classified as "non-progressive", grade 3 and 4 or grade increase as "progressive". All SSc patients were evaluated the items as used for 2013 ACR/EULAR criteria.

Results: The mean durations of follow-up period were $9.5 \pm 0.6$ years. The number of SSc patients in each SIC grade at the initial evaluation and the end of follow-up were as follows; grade 1: initial vs end; 23 vs 24 , grade $2: 20$ vs 9 , grade 3: 10 vs 16 , grade $4: 2$ vs 6 . The SIC change "progressive" was $40.0 \%$ of SSc patients. Positive correlation between the esophageal diameters and the SIC grade was observed in SSc patients at the initial evaluation $(r=0.61 p<0.01)$ and the end of follow-up $(r=0.71 p<0.01)$. The esophageal diameters at the initial evaluation were significantly wider in SIC "progressive" group than in "non-progressive" group (non-progressive vs progressive; $21.8 \pm 6.5$ vs $30.9 \pm 8.6 \mathrm{~mm}, \mathrm{p}<0.0001$ ). The frequencies of SSc patients with ACA-positive and sclerodactyly were higher but with puffy fingers were lower in SIC "progressive" group than in "non-progressive" group (non-progressive vs progressive; 24.2 vs $59.1 \%, p=0.009 ; 33.3$ vs $63.6 \%$, $\mathrm{p}=0.03 ; 72.7$ vs $45.5 \%, \mathrm{p}=0.03$ ). The prevalence of pitting scar and Interstitial lung disease were tended to be higher in SIC "progressive" compared to SIC "non-progressive" group (non-progressive vs progressive; 15.6 vs $36.4 \%, p=0.07$; 21.1 vs $45.5 \%, p=0.06$ )

Conclusions: The present study demonstrated that the progression of small intestinal involvement was associated with esophageal dilatation at the initial evaluation during long-term follow-up of SSc patients. Our findings also suggested that ACA and skin thickening of the fingers were important factors of small intestinal involvement in SSc patients. The SIC may be a useful tool for the assessment of Gl tract involvement in SSc patients during long-term follow-up.

Disclosure of Interest: None declared

DOI: 10.1136/annrheumdis-2017-eular.2353

\section{OP0128 DETECTION OF SUB-CLINICAL DIFFUSE MYOCARDIAL FIBROSIS BY NATIVE T1 MAPPING MAGNETIC RESONANCE IMAGING IN A PROSPECTIVE SYSTEMIC SCLEROSIS COHORT}

V. Poindron, E. Chatelus, M. Canuet, P. Germain, S. El Ghannudi, T. Martin. National Referral Center for Systemic Autoimmune Diseases, Hôpitaux Universitaires de Strasbourg, Strasbourg, France

Background: Cardiac involvement in systemic sclerosis (SSc) is the second most frequent SSc-related cause of death. It remains mostly asymptomatic in early stages and is underdiagnosed with routine non-invasive screening. Cardiac magnetic resonance imaging (CMR) is becoming a key actor as it has a better sensitivity than echocardiography (echo). CMR can detect diffuse myocardial fibrosis (DMF) by native T1 mapping, myocardial edema (ME) by T2 mapping and focal fibrosis by late gadolinium enhancement (LGE). Ntusi et al. reported an increase of $5 \%$ of $\mathrm{T} 1$ value suggestive of DMF in 10 of 19 SSc patients[1]

Objectives: To determine the prevalence of cardiac involvement by CMR native T1 and T2 mapping and its correlation with echo data and non-cardiac manifestations in SSc patients.

Methods: 72 patients fulfilling ACR/EULAR classification criteria were prospectively included between 2013-2016. They underwent CMR at 1.5T, including native T1 and T2 mapping, and LGE. Normal T1 value determined in our center was $1032 \pm 39 \mathrm{msec}$. In the present study an elevated native T1>1082 msec was likely representing ventricle (especially left) DMF and T2 $>55$ msec representing $\mathrm{ME}$ [2].

Results: Patients characteristics: mean age: $56 \pm 14.8$; diffuse disease: 38 (52.8\%); anti-Scl70 positivity: 29 (40.3\%); anti-RNApollII positivity: 6 (8.3\%); 21 $(29.2 \%)$ patients had early disease ( $<2$ years from first non-Raynaud symptom). The mean T1 was $1064 \pm 41.6 \mathrm{msec}$ and T2 was $51.8 \pm 2.9 \mathrm{msec}$. 36 patients $(50 \%)$ had DMF but only $6(8.3 \%)$ had ME. The mean T1 in DMF cases was $1097 \pm 14$, and the T2 in ME cases was $58.2 \pm 1.6 \mathrm{msec}$. LGE was reported in $25.7 \%$ of patients. Although LGE was more frequent in patients with DMF than in those without DMF (13 vs $5, p=0.024)$, only $13(36.1 \%)$ DMF patients had LGE. Left ventricular ejection fraction (L-VEF), left ventricular telediastolic volume (L-VTDV), Right-VEF and Right-VTDV were similar in DMF and non-DMF (N-DMF) groups. Echo was normal in $18(50 \%)$ patients with DMF and in 25 $(69.4 \%$ ) of N-DMF group ( $\mathrm{p}=0.09$ ). DMF and N-DMF groups were similar for sex ratio, age, cardiovascular risk factors and ischemic heart disease. DMF was more frequent in patients with late disease ( 27 vs $9, p=0.05$ ). T1 value was positively correlated to pulmonary arterial hypertension and digital ulcerations together $(r=0.31, p=0.008)$ but not with Rodnan skin score. Six patients $(8.3 \%)$ died during the inclusion period: 5 were in DMF group $(p=0.09)$. The alterations of L-VEF and R-VEF were correlated $(r=0.45, p=0.009)$. DMF was not associated with skin subsets, interstitial lung disease, auto-antibody profile, all echo parameters, CRP and BNP.

Conclusions: Native T1 mapping detects left ventricular DMF in $50 \%$ of patients with SSc including $43 \%(9 / 21)$ of the patients with early disease. Among them, $36 \%$ had normal echocardiography and CMR L-VEF and no LGE.

\section{References:}

[1] Ntusi NA, Piechnik SK, Francis JM, et al. Subclinical myocardial inflammation and diffuse fibrosis are common in systemic sclerosis a clinical study using myocardial T1-mapping and extracellular volume quantification.J Cardiovasc Magn Reson 2014;16:21.

[2] Germain P, El Ghannudi S, Jeung MY et al. Native T1 mapping of the heart a pictorial review. Clin Med Insights Cardiol 2014;8:1

Disclosure of Interest: None declared

DOI: 10.1136/annrheumdis-2017-eular.4955

\section{OP0129 MYCOPHENOLATE MOFETIL VERSUS CYCLOPHOSPHAMIDE IN SCLERODERMA-RELATED INTERSTITIAL LUNG DISEASE IN A REAL LIFE SCENARIO}

${ }_{\text {H. Fretheim }}{ }^{1}, \varnothing$. Midtvedt ${ }^{1}$, E. Volkmann ${ }^{2}$, T. Garen ${ }^{1}$, M.B. Lund ${ }^{1}$,

T. Aaløkken ${ }^{1}, \varnothing$. Molberg ${ }^{1}$, A.-M. Hoffmann-Vold ${ }^{1}$. ${ }^{1}$ Oslo University Hospital, Oslo, Norway; ${ }^{2}$ UCLA, LA, United States

Background: Systemic sclerosis (SSc) is a serious disease with high risk of interstitial lung disease (ILD); a feared complication with poor prognosis. The SLS $2^{1}$ supported the clinical effectiveness of Mycophenolate Mofetil (MMF) and Cyclophosphamide (Cyc).

Objectives: Evaluate the effect of MMF and Cyc on SSc-ILD in a population based cohort.

Methods: All SSc patients at Oslo University Hospital (OUH) are included in an prospective, observational SSc cohort. All patients are followed annually by rheumatologists and data are recorded in the Norwegian systemic connective tissue disease registry (NOSVAR). Here, we assessed patients diagnosed after the year $2000(n=433)$. Longitudinal pulmonary function tests (PFTs), HRCT lung image results, pulmonary hypertension $(\mathrm{PH})$, clinical and demographic data, antibodies and vital status were obtained from NOSVAR. Outcome measures were vital status in December 2016, extent of fibrosis measured on HRCT, FVC and DLCO development.

Results: Among 262 patients with complete data on treatment and ILD, $21(8 \%)$ patients received treatment with Cyc, $14(5.3 \%)$ with MMF and $17(6.5 \%)$ with combination therapy of MMF and Cyc. $55.7 \%$ of patients did not receive any treatment for ILD. Baseline characteristics, lung fibrosis and function did not differ significantly between the MMF and Cyc (Table 1). Patients treated with Cyc show a trend of higher mortality. Treatment with Cyc resulted in a significant improvement of lung fibrosis; whereas treatment with MMF resulted in significant progression in lung fibrosis $(-1.3 \%$ (SD 9.3) and $7.5 \%$ (SD 11.1), $\mathrm{p}=0.024$ ) (Table 1). MMF treatment showed a significant improvement in the DLCO\% (1.8\% [SD 4.6]); whereas Cyc had a significant decline in the DLCO\% $(-2.1 \%$ [SD 3.5], $p=0.14)$. Less patients treated with MMF developed $\mathrm{PH}$ compared with Cyc treatment (1[7.7\%] compared to $8[42.1 \%], p=0.038$ ) (Table 1).

Table 1. Demographics and clinical characteristics of the OUH cohort

\begin{tabular}{lcccc}
\hline & Total cohort $(\mathrm{n}=262)$ & Cyc $(\mathrm{n}=21)$ & MMF $(\mathrm{n}=14)$ & $\mathrm{p}$-value \\
\hline Age at diagnosis, yrs (SD) & $51.4(15.5)$ & $52.6(12.1)$ & $43.5(18.5)$ & - \\
Follow-up period, yrs (SD) & $6.4(4.0)$ & $5.5(3.9)$ & $4.1(2.3)$ & - \\
Male, $\mathrm{n}(\%)$ & $42(16.0)$ & $6(28.6)$ & $2(14.3)$ & - \\
dcSSc, $\mathrm{n}(\%)$ & $68(26.0)$ & $8(38.1)$ & $10(71.4)$ & - \\
Deceased, $\mathrm{n}(\%)$ & $54(20.6)$ & $8(38.1)$ & $4(28.6)$ & - \\
PH, $\mathrm{n}(\%)$ & $35(14)$ & $8(42.1)$ & $1(7.7)$ & 0.038 \\
BL FVC\%,(SD) & $96.4(19.5)$ & $83.8(16.5)$ & $81.5(13.3)$ & - \\
BL DLCO\%,(SD) & $70.4(19.5)$ & $54.2(16.2)$ & $61.8(14.9)$ & - \\
BL fibrosis\%,(SD) & $6.1(11.3)$ & $19.1(15.8)$ & $10.8(10.3)$ & - \\
FVC\% decline,(SD) & $-0.9(14.4)$ & $-3.3(14.3)$ & $2.6(11.3)$ & - \\
Annual FVC\% decline,(SD) & $-0.02(6.1)$ & $-0.3(9.1)$ & $2.3(4.1)$ & - \\
DLCO\% decline,(SD) & $-9.1(13.5)$ & $-9(14.1)$ & $0.7(12.4)$ & - \\
Annual DLCO\% decline,(SD) & $-2(5.1)$ & $-2.1(3.5)$ & $1.8(4.6)$ & 0.014 \\
Fibrosis\% progression,(SD) & $1.2(5.7)$ & $-1.3(9.3)$ & $7.5(11.1)$ & 0.024 \\
Annual fibrosis\% progression,(SD) & $0.5(2.8)$ & $-0.3(4.9)$ & $2.3(4.7)$ & - \\
\hline
\end{tabular}

n: number; BL:baseline, SD: standard deviation; FVC: Forced vital capacity; DLCO: diffusing lung capacity for carbon monoxide; dcSSc: diffuse cutaneous SSc; PH: pulmonary hypertension.

Conclusions: Preliminary data from our population based cohort indicate that in a real-life scenario treatment effects of Cyc and MMF appear comparable to randomized clinical trials, but there are some potentially important nuances. Cyc seems to halt fibrosis progression, but toxicity is a major concern, while MMF could have effects on DLCO decline and the development of $\mathrm{PH}$.

References:

[1] Tashkin DP et al. Mycophenolate mofetil versus oral cyclophosphamide in SLS II. Lancet Respir Med. 2016.

Disclosure of Interest: None declared

DOI: 10.1136/annrheumdis-2017-eular.4819 